\title{
Experimental Study on Ferromagnetic Shunt Effects on the Critical Current of BSCCO Tape in Stacked Conductors
}

\author{
J. Sun, ${ }^{1}$ H. Ohara, ${ }^{2}$ and S. Yamaguchi ${ }^{1,2}$ \\ ${ }^{1}$ Center of Applied Superconductivity and Sustainable Energy Research (CASER), Chubu University, 1200 Matsumoto-cho, Kasugai, \\ Aichi 487-8501, Japan \\ ${ }^{2}$ Department of Electrical Engineering, Chubu University, 1200 Matsumoto-cho, Kasugai, Aichi 487-8501, Japan \\ Correspondence should be addressed to J. Sun; j_sun@isc.chubu.ac.jp
}

Received 12 December 2016; Accepted 24 January 2017; Published 27 February 2017

Academic Editor: Sergei Sergeenkov

Copyright (C) 2017 J. Sun et al. This is an open access article distributed under the Creative Commons Attribution License, which permits unrestricted use, distribution, and reproduction in any medium, provided the original work is properly cited.

\begin{abstract}
In the $200 \mathrm{~m}$ high temperature superconducting (HTS) cable test facility at Chubu University constructed in 2010, a three-layer structure of the tapes in the cable is employed for obtaining the high current capacity up to $2 \mathrm{kA}$. Previous study shows that the critical current is affected by the layout of the tapes such as gaps and the current feeding mode. In the stacked tape conductors, the critical current of BSCCO tapes shows strong dependence on the current feeding directions between the tapes. The critical current is improved when the opposite-direction current is applied to them and degraded for the same-direction current feeding mode because of strong magnetic field interaction between them from the transport current in each tape. This paper presents the measurements of the critical currents of a BSCCO tape in the stacked conductors with the ferromagnetic materials. By using the ferromagnetic materials surrounding the tapes, self-field of HTS tapes is affected and their critical currents are improved by more than 10\%. Ferromagnetic shunt effects on the critical current of HTS tape are reported through the magnetic field analysis.
\end{abstract}

\section{Introduction}

Since the first DC high temperature superconducting (HTS) power cable test facility was constructed at Chubu University [1], studies and tests on the DC HTS power cable have been continued and a $200 \mathrm{~m}$ DC HTS cable system, called CASERII, was set up successfully in 2010 [2]. Figure 1(a) shows a photo of the CASER-II's cable. A three-layer coaxial structure of the cable is used as in Figure 1(b). In order to improve the cable property by the tape arrangements, the performance of the HTS tapes for the DC power cable applications was investigated through the critical current measurements [3].

To obtain a high current density of a cable, a multilayer structure is needed and extremely stacked tape conductors are one of the choices to make this by using multilayer conductors as in Figure 1. The stacked structure makes the conductor compact but increases the magnetic field interaction between them. The latter would significantly affect the performance of HTS tapes in the stacked conductor. In a previous study, the critical current of BSCCO tape in the three-layer stacked conductors is dependent on current feeding mode [4]. With the opposite-direction current applied to each tape in the three-layer stacked conductors, the critical current of middle tape increases sharply by $50 \%$ due to the effects on self-field from the currents in the neighboring tapes compared to that of single one. However, the critical current degrades for the same-direction current feeding because of effects on self-field from the neighboring current in the adjacent tapes.

FMs have been used to improve the performance of BSCCO tape by covering its edges [5] and HTS cable by covering the gap between the tapes as a diverter [6]. However, the increase in the critical current is less than $7 \%$ in [5] by covering thin Ni coating layer $(24 \mu \mathrm{m})$ on edges of a BSCCO tape. In this work, we investigated the critical current of BSSCO tape in the stacked conductors covered with and without Ferromagnetic materials (FMs) by varying current feeding mode and the critical current can be improved by more than $10 \%$ experimentally. The insulated tapes are arranged in a tape-on-tape structure and different currents 


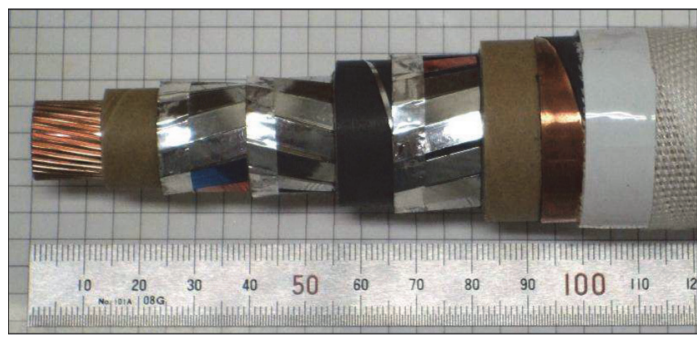

(a)

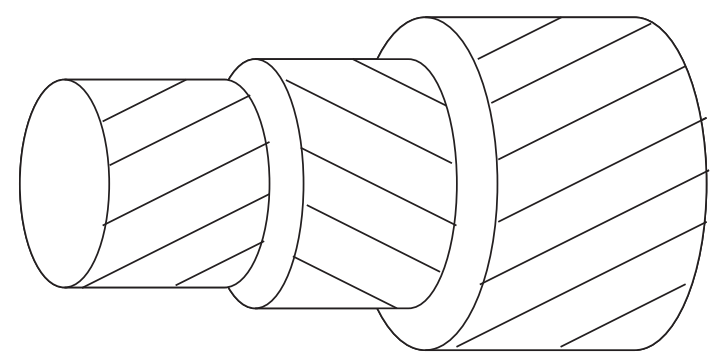

(b)

FIGURE 1: (a) A photo of the CASER-II power cable. (b) A schematic layout of the coaxial three-layer HTS conductor for the CASER-II cable.

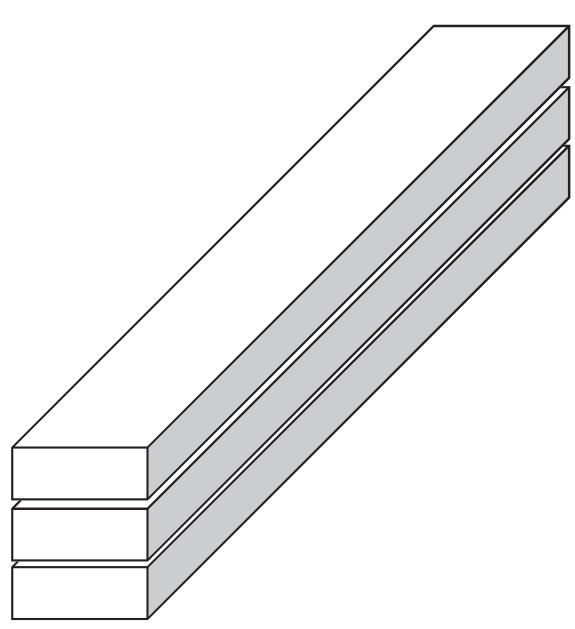

(a)

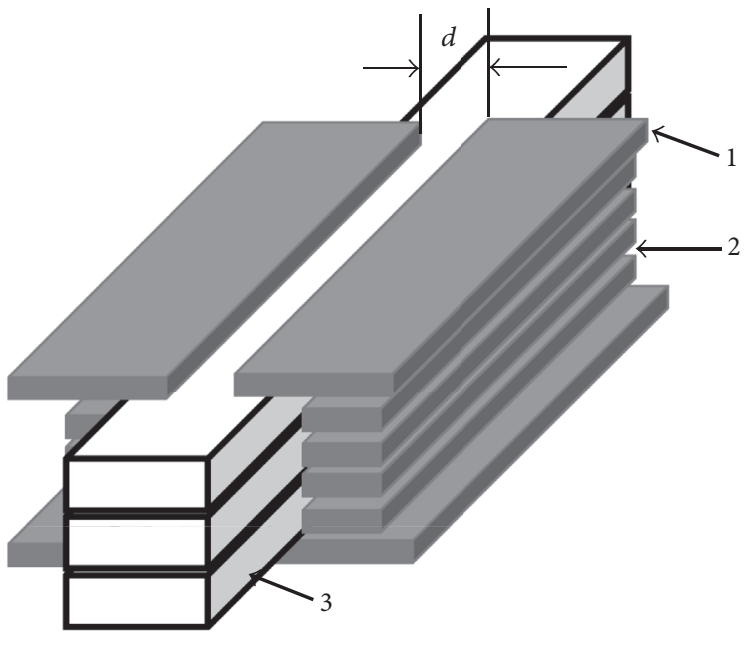

(b)

FIGURE 2: A schematic layout of three-layer stacked conductor without (a) and with (b) ferromagnetic shunt materials. 1: four pieces of 5 mm width ferromagnetic sheets; 2: eight pieces of $3 \mathrm{~mm}$ width ferromagnetic sheets; 3 : three HTS tapes; and $d$ : a space gap between $5 \mathrm{~mm}$ width ferromagnetic sheets above tape wide surface.

are applied to each tape. The critical current of the target sample is measured by the four-probe method by immersing the samples into a liquid nitrogen bath. We will present the experimental results and analysis on the magnetic field interactions between tapes to improve the superconductivity for DC power cable applications.

\section{Experiments}

The experimental setup of the critical current measurement is similar to previous report [3]. The HTS tapes are BSCCO tapes (Bi-2223) from Sumitomo Electric Industries. Three insulated BSCCO tapes (Type HT-CA) are used in the experiment with a cross section of $4.5 \mathrm{~mm} \times 0.35 \mathrm{~mm}$ and a length of $\sim 270 \mathrm{~mm}$ [7]. The critical current of BSCCO tape is $200 \mathrm{~A}$ in self-field at $77 \mathrm{~K}$. The tapes are arranged as a stacked structure as shown in Figure 2(a). Three voltage taps are soldered on each tape with the distance of 8 and $10 \mathrm{~cm}$. The ferromagnetic sheets have a thickness of $0.3 \mathrm{~mm}$. Eight $3 \mathrm{~mm}$ width FM sheets and four $5 \mathrm{~mm}$ width FM sheets are covered at edges with a space gap of $d$ as in Figure 2(b). The ferromagnetic materials are provided from JFE steel [8].
Two power supplies (PR10-300) with output current/ voltage $300 \mathrm{~A} / 10 \mathrm{~V}$ are used to control the current feeding mode in the three-layer stacked conductor as in Figures 3(a) and 3(b). The first power supply $\left(I_{1}\right)$ is used for the current feeding to the middle BSCCO tape 2 and the second power supply $\left(I_{2}\right)$ for bottom and upper BSCCO tapes (1 and 3) in series. The transport current is measured by a current shunt resistor $0.25 \mathrm{~m} \Omega$. All voltage signals are measured by a Keithley 2700 multimeter with sensitivity of $1 \mu \mathrm{V}$. The $I-V$ characteristic curves are measured through the standard four-probe method at liquid nitrogen temperature of $77 \mathrm{~K}$.

\section{Results and Discussion}

Figures 4(a) and 4(b) present comparisons of E-I curves of a BSCCO tape for single- and three-layer stacked tape conductor for different neighboring current of $\pm 160 \mathrm{~A}$ without and with FMs, respectively. The electric fields $(E)$ are obtained by normalizing the voltages to the distance between the voltage taps. The significant difference between the threelayer stacked conductors and single one is shown when the currents in the adjacent tapes are changed. In the case of no 


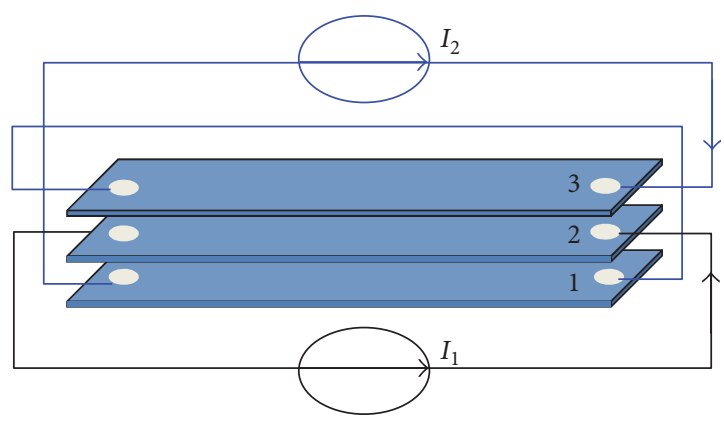

(a)

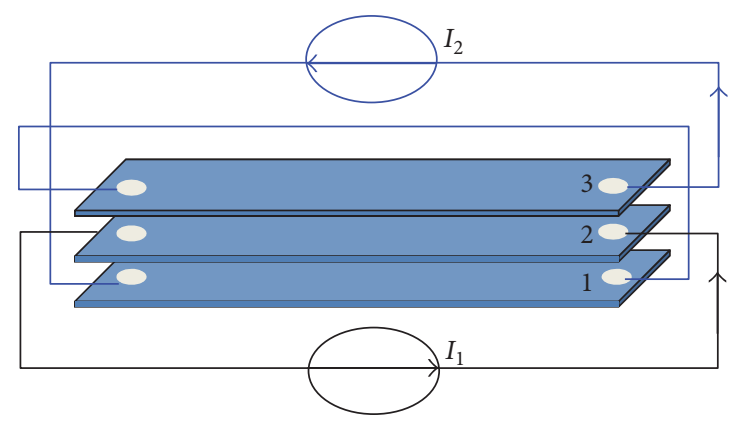

(b)

FIgURE 3: Schemes of (a) same-direction $(\uparrow \uparrow \uparrow)$ and (b) opposite-direction $(\downarrow \uparrow \downarrow)$ current feeding modes. The transport current applied to tape 1 and tape 3 is controlled by power supply $\left(I_{2}\right)$ and the transport current for tape 2 is controlled by $I_{1}$. The critical current of tape 2 is measured by four-probe method.

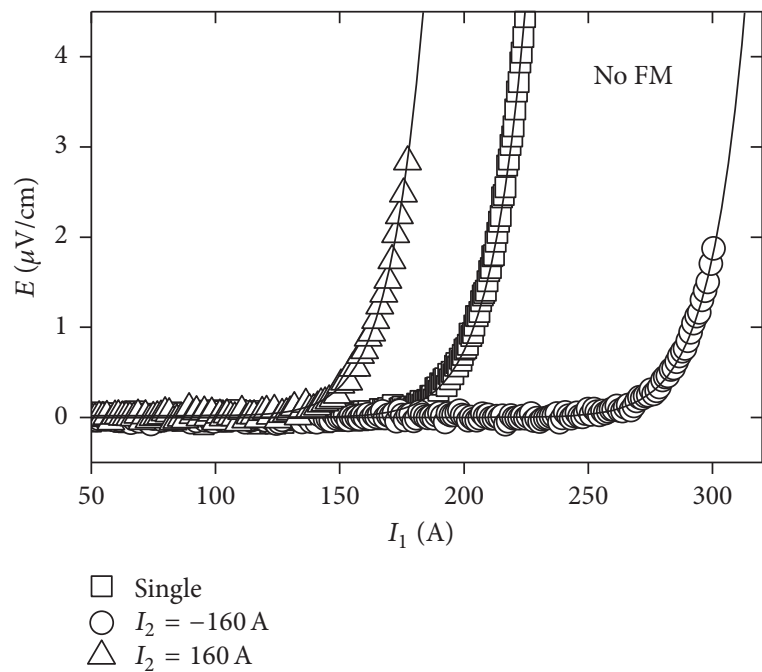

(a)

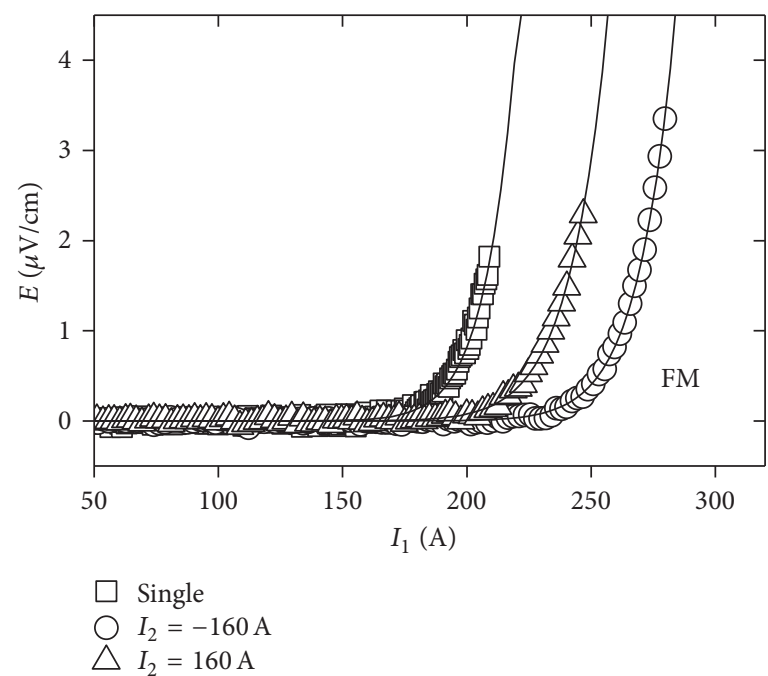

(b)

FIGURE 4: $E-I$ curves of BSCCO tape for single ( $\square$ ) and stacked conductors $(\bigcirc$ and $\triangle$ ) with different currents of $\pm 160 \mathrm{~A}$ applied to the adjacent BSCCO tapes without (a) and with (b) FM.

FM, the $I-V$ curves shift up for opposite current of -160 A and down for the same current of $160 \mathrm{~A}$, respectively. When FMs are used to cover at the edges of the stacked conductor, improvement of transport properties can be seen from $E-I$ curves in Figure 4(b). To obtain the critical current, we fit the $E$-I curves by the following equation:

$$
E=E_{0} \times\left(\frac{I}{I_{c}}\right)^{n}
$$

where $E$ is electric field, $E_{0}$ is electric field criterion of $1 \mu \mathrm{V} / \mathrm{cm}, I$ is transport current, $I_{c}$ is critical current, and $n$ is $n$-value, respectively.

Figure 5 shows the measured critical currents of BSCCO tape with respect to neighboring current without FMs. The horizontal axis is the neighboring current $I_{2}$ in the adjacent tapes 1 and 3 as in Figure 3. The positive current is the samedirection current ( $\uparrow \uparrow$ ) as in Figure 3(a) and the negative current is the opposite-direction current $(\downarrow \uparrow \downarrow)$ as in Figure 3(b).
The critical current of single tape $\left(I_{c 0}\right)$ is measured to be $201 \mathrm{~A}$. When the positive currents are applied to the stacked conductor, the critical current of the middle tape is improved significantly. When the neighboring current becomes more than $80 \mathrm{~A}$, the critical current degrades sharply by $20 \%$ as in Figure 5(b).

Figure 6 shows the measured $n$-value of BSCCO tapes in the stacked conductor without FMs in comparison with a single tape. The $n$-value shows similar trend with respect to the neighboring current as in Figure 5.

Figures 7 and 8 present the measured critical current and $n$-value of BSCCO tape in the stacked conductor with FMs as in Figure 2(b). The critical current increases with increasing the neighboring current $I_{2}$ for the opposite-direction current and decrease with the neighboring current $I_{2}$ for the samedirection current. The $n$-value shows similar behavior as the critical current with respect to the neighboring current.

Dependence of the critical current on the neighboring current is different for the stacked conductor covered with or 


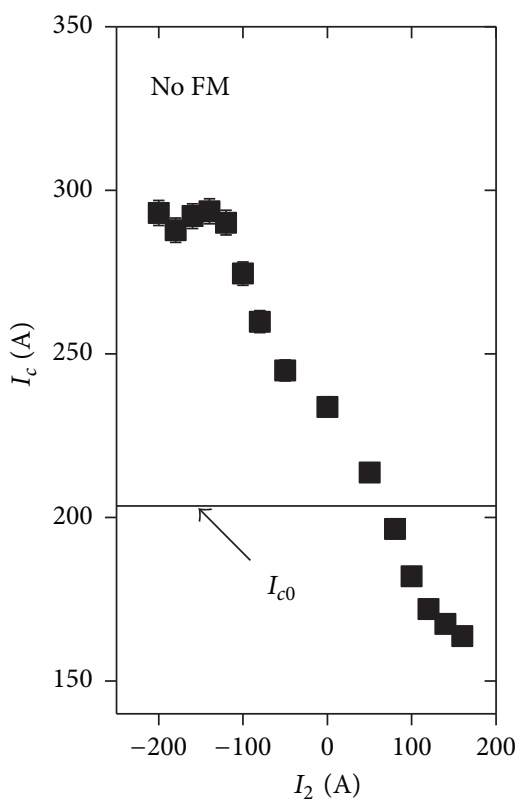

(a)

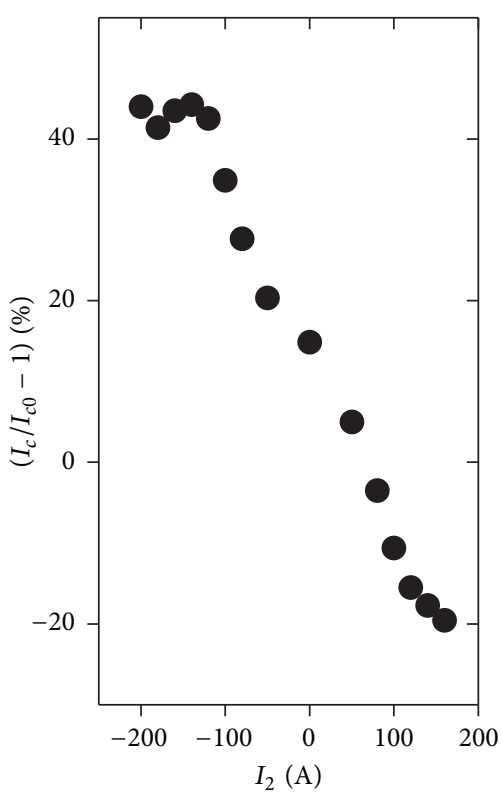

(b)

FIGURE 5: (a) $I_{c}$ versus the applied current to the neighboring tapes and (b) increase of $I_{c}$ with respect to the neighboring current for three-layer stacked conductor without FM.

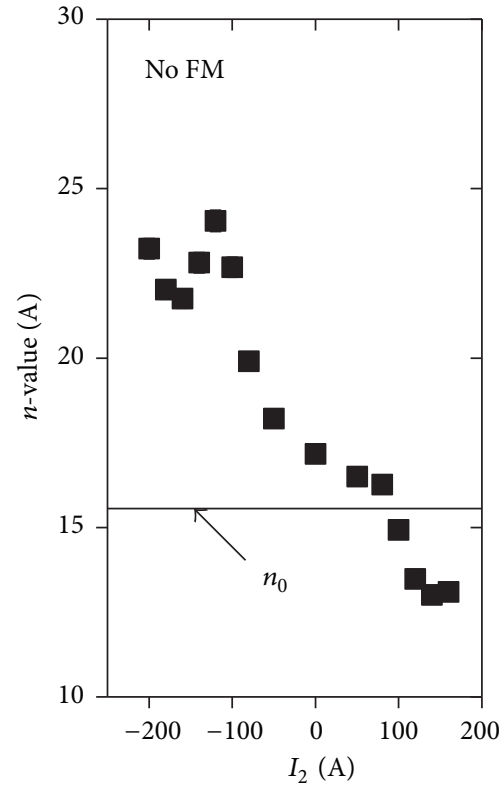

(a)

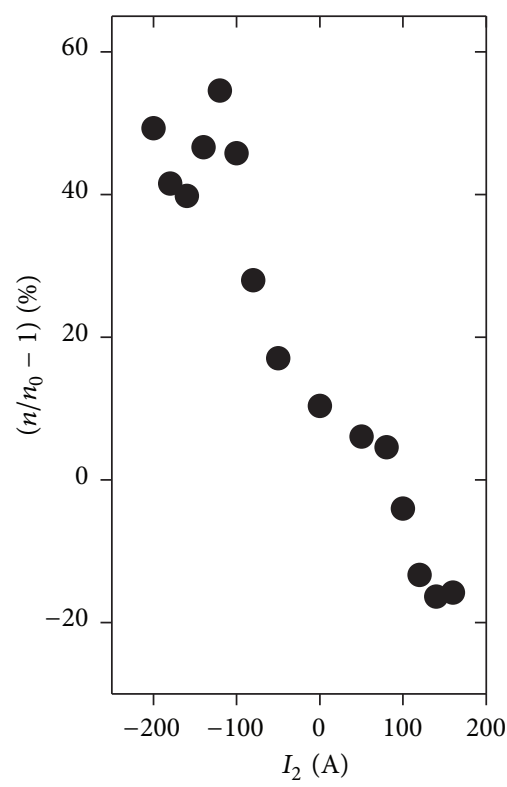

(b)

Figure 6: (a) $n$-value versus the applied current to the neighboring tapes and (b) increase of $n$ with respect to the neighboring current for three-layer stacked conductor without FM.

without FMs. When the current of the same direction $(\uparrow \uparrow \uparrow)$ is applied to each tape, the critical current declines without FMs, which is similar with the previous report [4]. However, the critical current is improved by covering the edges of the stacked conductor by FMs as in Figures 7 and 8.
The magnetic field simulation is performed by the finite element method (FEM) ANSYS code [9] to analyze the ferromagnetic shunt effects on the self-field. Figure 9 presents the magnetic flux distribution for three-layer stacked conductor with and without FMs under the same-direction 


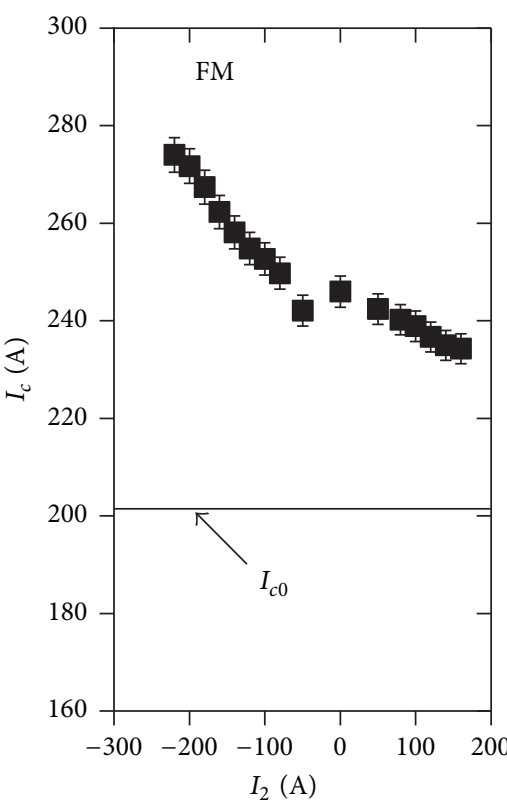

(a)

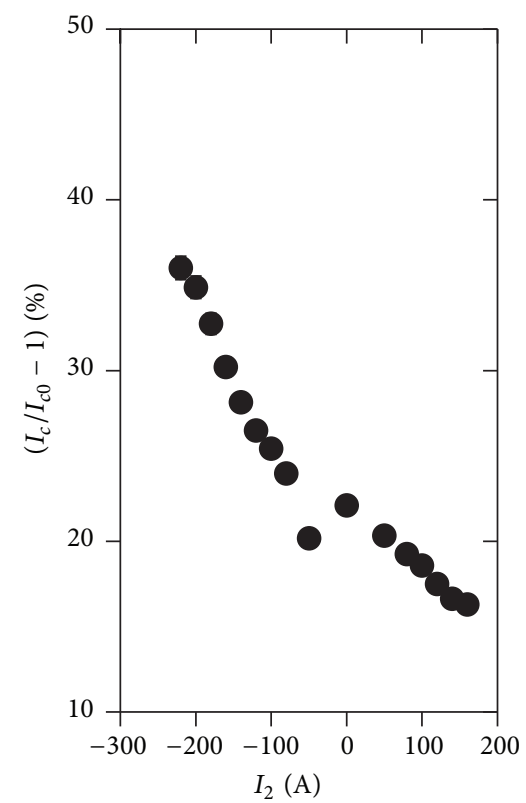

(b)

FIGURE 7: (a) $I_{c}$ versus the applied current to the neighboring tapes and (b) increase of $I_{c}$ with respect to the neighboring current for three-layer stacked conductor with FMs.

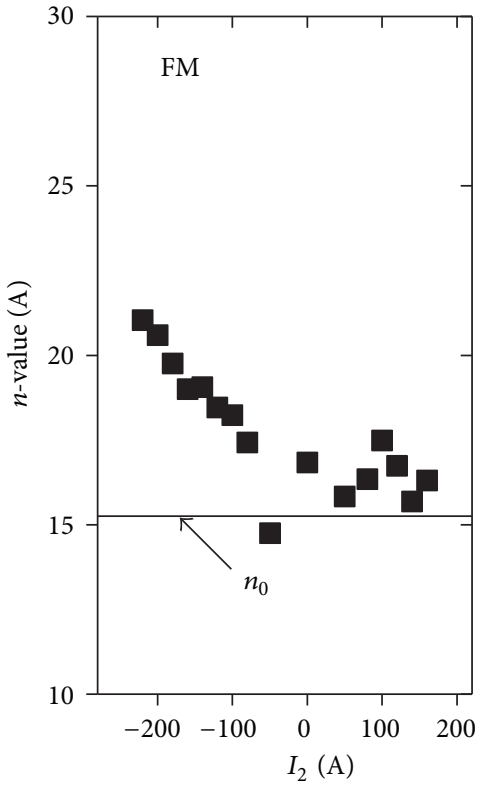

(a)

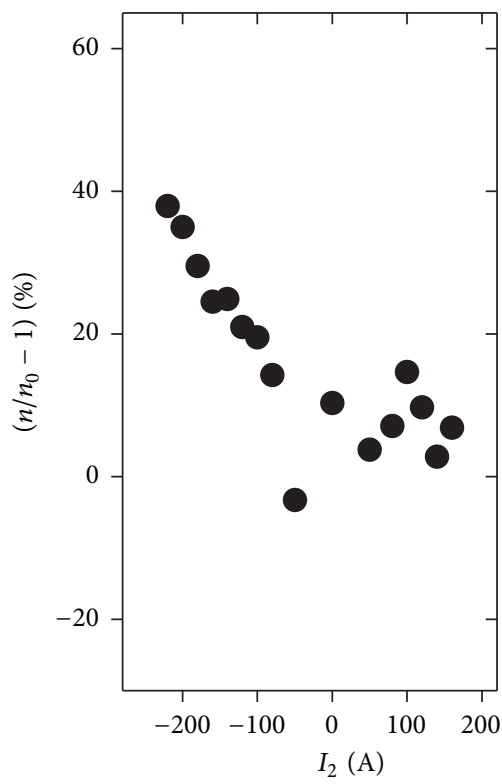

(b)

FiguRE 8: (a) $n$-value versus the applied current to the neighboring tapes and (b) increase of $n$-value with respect to the neighboring current for three-layer stacked conductor with FMs.

current $(\uparrow \uparrow \uparrow)$ of $200 \mathrm{~A}$. Figure 10 presents the magnetic flux distribution for opposite-direction current of $200 \mathrm{~A}(\uparrow \downarrow \uparrow)$. The current of $200 \mathrm{~A}$ is assumed to be uniformly distributed in the HTS tapes. The nonlinear magnetization $B-H$ relations of the ferromagnetic materials are used in the calculations [8].

Compared with the magnetic field for no-FM covers, the magnetic flux is attracted through the FMs and leads to reduction of the magnetic field in the middle tape for the same-direction current as in Figure 9. However, as shown in Figure 9(b), the magnetic fields around the middle tape become neutral from neighboring current with FMs covered at the edges, which results in the great improvement of the critical current as above observed in Figures 7 and 8 . In the case of the opposite-direction current, the magnetic flux 


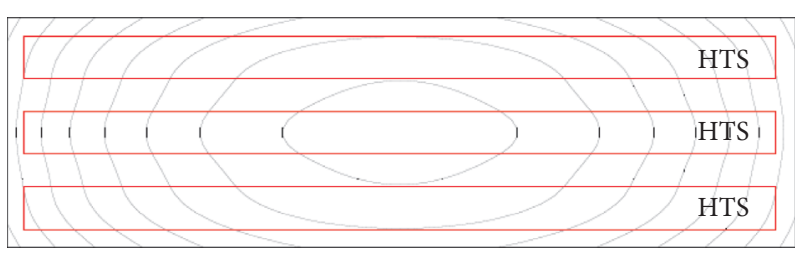

(a)

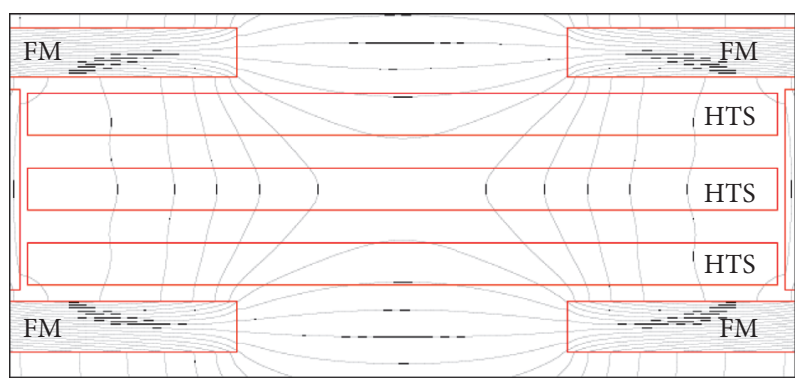

(b)

FIGURE 9: Calculated magnetic flux lines by finite element method ANSYS code [9] for three-layer stacked tape conductors (a) without FMs and (b) with FMs at the edges under the same-direction ( $\uparrow \uparrow)$ current feeding mode. The currents in each tape are $I_{1}=I_{2}=I_{3}=$ $200 \mathrm{~A}$ as in Figure 3(a).

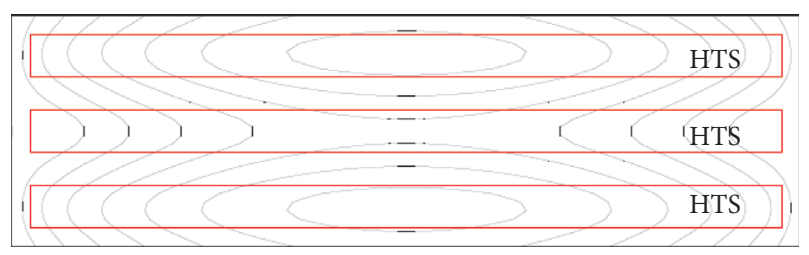

(a)

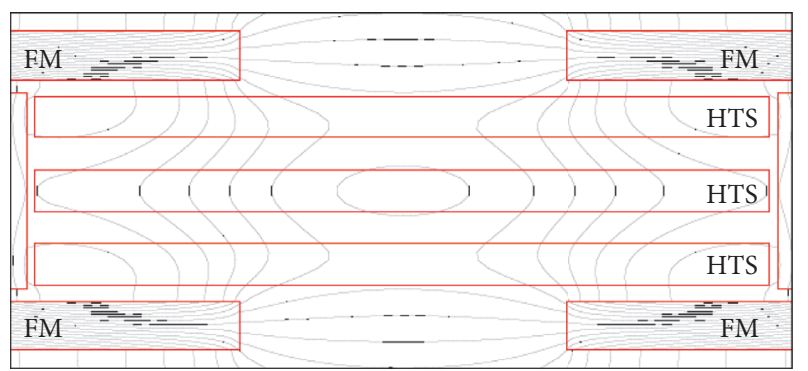

(b)

FIGURE 10: Calculated magnetic flux lines by finite element method ANSYS code [9] for three-layer stacked tape conductors (a) without FMLs and (b) with FMs at the edges under the antidirection $(\uparrow \downarrow \uparrow)$ current feeding mode. The currents in each tape are $I_{1}=-I_{2}=I_{3}=$ $200 \mathrm{~A}$ as in Figure 3(b).

is also absorbed by the FMs as in Figure 10(b); however, the magnetic flux distribution of stacked conductor with or without FMs is similar as in Figure 10, which leads to no improvements of the critical current or $n$-value as in Figures 5 and 6.

\section{Conclusion}

We investigated the critical current and $n$-value of a $200 \mathrm{~A}$ level BSSCO tape in the stacked conductors covered with and without ferromagnetic materials by varying current feeding mode. With the opposite-direction $(\uparrow \downarrow \uparrow)$ current applied to each tape in the three-layer stacked tape conductors, both the critical currents and the $n$-values of middle tape are improved due to the effects on the self-field of the magnetic field from the neighboring current. Prominent improvement of the critical current by covering FMs at the edges around the stacked conductor comparing with uncovered one is observed when the same-direction ( $\uparrow \uparrow \uparrow)$ current mode is applied due to the ferromagnetic shunt effects through the magnetic field calculations by FEM.

\section{Competing Interests}

The authors declare that they have no competing interests.

\section{Acknowledgments}

The authors acknowledge all members in CASER, Chubu University, for their useful discussion. The authors also acknowledge Professor A. Iiyoshi, the Board Chairperson of Chubu University, for his continuous encouragements through the work. The authors acknowledge Dr. Hiroshi Fujiwara, a Professor of Keio University and the President of Nano-Opt Energy Corporation, for his financial support on the construction of the CASER-II. Parts of this work were supported by a grant of Strategic Research Foundation GrantAided Project for Private Universities Ministry of Education, Culture, Sports, Science and Technology (MEXT).

\section{References}

[1] S. Yamaguchi, M. Hamabe, I. Yamamoto et al., "Research activities of DC superconducting power transmission line in Chubu University," Journal of Physics: Conference Series, vol. 97, no. 1, Article ID 012290, 2008.

[2] S. Yamaguchi, T. Kawahara, M. Hamabe et al., "Design and construction of 200meter high temperature superconducting DC power cable test facility in Chubu University," in Proceedings of the 23rd International Cryogenic Engineering Conference and International Cryogenic Materials Conference (ICEC-ICMC '10), pp. 1041-1047, Wroclaw, Poland, 2011.

[3] J. Sun, S. Yamauchi, M. Sugino et al., "Critical current measurements for design of superconducting DC transmission power cable," Physica C: Superconductivity and its Applications, vol. 471, no. 21, pp. 1313-1316, 2011.

[4] J. Sun, H. Watanabe, M. Hamabe, N. Yamamoto, T. Kawahara, and S. Yamaguchi, "Critical current behavior of a BSCCO tape in the stacked conductors under different current feeding mode," Physica C: Superconductivity, vol. 494, pp. 297-301, 2013.

[5] S. Safran, M. Vojenciak, A. Gencer, and F. Gömöry, "Critical current and AC loss of DI-BSCCO tape modified by the deposition of ferromagnetic layer on edges," IEEE Transactions on Applied Superconductivity, vol. 20, no. 5, pp. 2294-2300, 2010.

[6] M. Vojenčiak, J. Šouc, and F. Gömöry, "Critical current and AC loss analysis of a superconducting power transmission 
cable with ferromagnetic diverters," Superconductor Science and Technology, vol. 24, no. 7, Article ID 075001, 2011.

[7] N. Ayai, K. Yamazaki, M. Kikuchi et al., "Electrical and mechanical properties of DI-BSCCO Type HT reinforced with metallic sheathes," IEEE Transactions on Applied Superconductivity, vol. 19, no. 3, pp. 3014-3017, 2009.

[8] S. E. Zirka, Y. I. Moroz, P. Marketos, A. J. Moses, D. C. Jiles, and T. Matsuo, "Generalization of the classical method for calculating dynamic hysteresis loops in grain-oriented electrical steels," IEEE Transactions on Magnetics, vol. 44, no. 9, pp. 21132126, 2008.

[9] ANSYS $^{\circledR}$, Academic Teaching Advanced, Release 12.1, ANSYS Inc., http://www.ansys.com. 

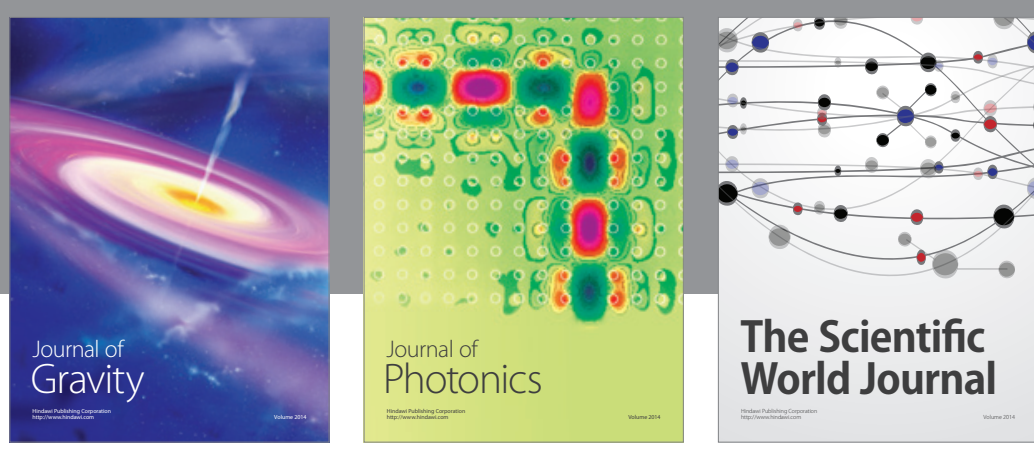

The Scientific World Journal
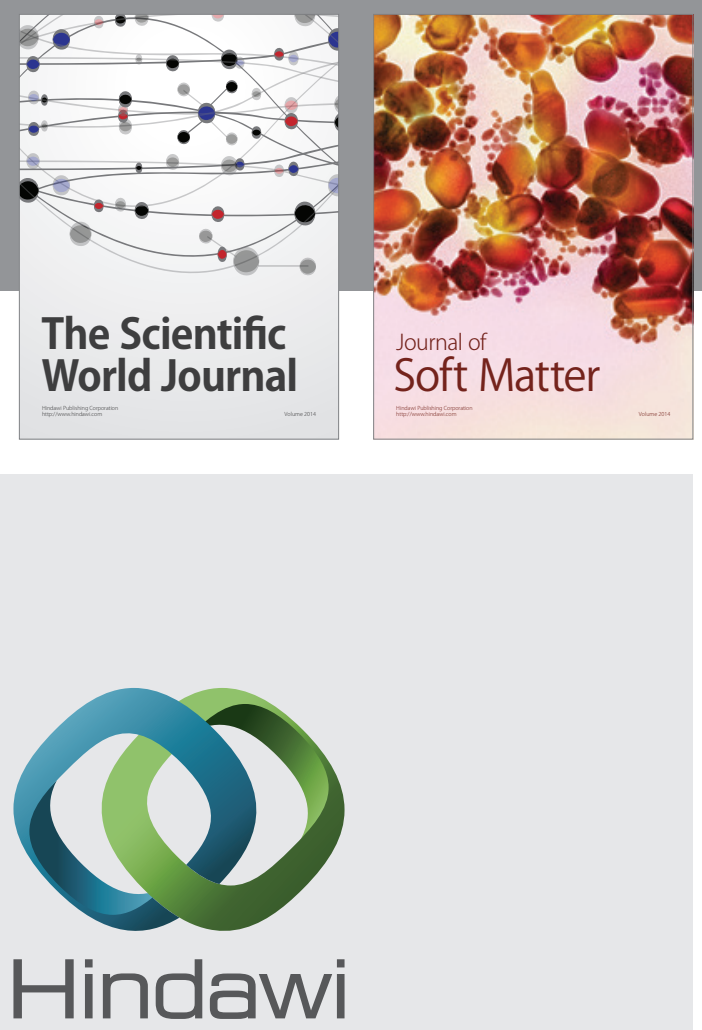

Submit your manuscripts at

https://www.hindawi.com
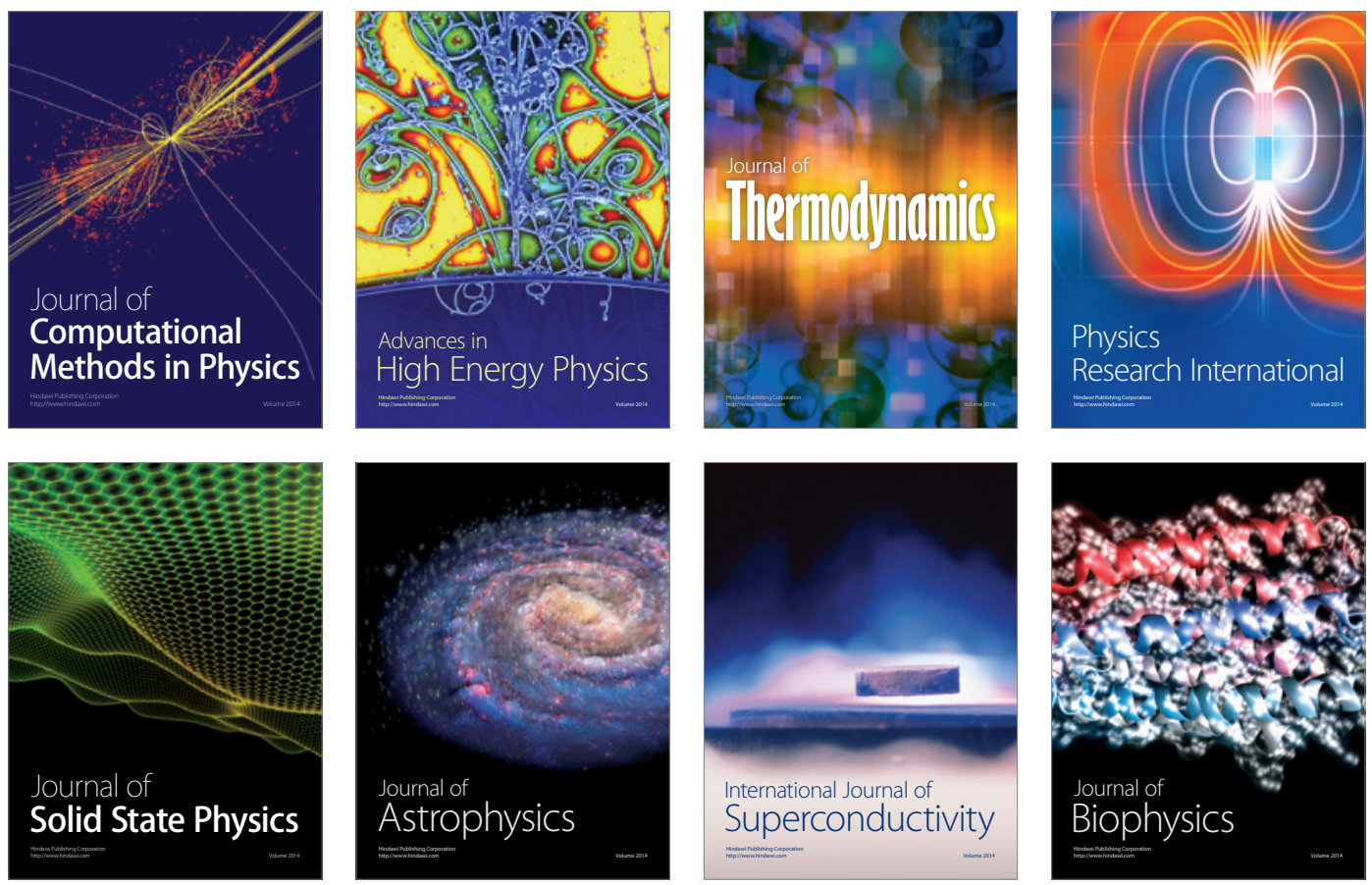
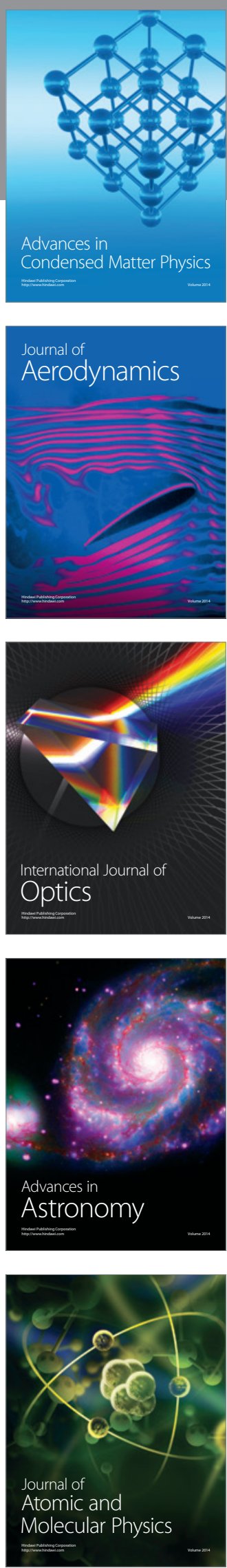